\title{
Local time variation of the amplitude of geomagnetic sudden commencements (SC) and SC-associated polar cap potential
}

\author{
T. Araki ${ }^{1}$, S. Tsunomura ${ }^{2}$, and T. Kikuchi ${ }^{3}$ \\ ${ }^{1}$ SOA Key Laboratory for Polar Science, Polar Research Institute of China, Shanghai, China \\ ${ }^{2}$ Japan Meteorological Agency, Tokyo, Japan \\ ${ }^{3}$ Solar Terrestrial Environmental Laboratory, Nagoya University, Nagoya, Japan
}

(Received February 11, 2009; Revised March 16, 2009; Accepted March 21, 2009; Online published April 8, 2009)

\begin{abstract}
Local time (LT) variations of the averaged $H$-component amplitude of geomagnetic sudden commencements (SCs) observed at Memambetsu (geomagnetic latitude $35.4^{\circ}$ ) are derived separately for the summer and winter season. The amplitude was found to be higher at nighttime than daytime in both seasons, and the LT variations showed a good agreement with calculated LT variations of the resultant magnetic field due to a pair of fieldaligned currents (FACs) and ionospheric currents produced by the FACs. The DL- and DP-component of the disturbance field of SC can be separated based on a comparison of the observed and calculated LT variations. A method is introduced by which we can estimate the potential voltage difference associated with the FACs in the polar cap.
\end{abstract}

Key words: Geomagnetic sudden commencement (SC), LT variation, field-aligned current, ionospheric current, interplanetary shock.

\section{Introduction}

According to the physical model of geomagnetic sudden commencements (SCs) proposed by Araki (1977, 1994), the disturbance field of SC, Dsc, is given by

$$
\mathrm{Dsc}=\mathrm{DPpi}+\mathrm{DLmi}+\mathrm{DPmi}
$$

where pi and mi express the 'preliminary impulse (PI)' and 'main impulse (MI)', respectively. The DL-field is dominant at low latitudes and is produced mainly by the magnetopause current enhanced after impinging of the interplanetary shock or discontinuity on the magnetosphere. The DPpi- and DPmi-fields, which are dominant at high latitudes, form an adjacent two pulse structure with opposite sense and are produced by a pair of field-aligned currents (FACs) and ionospheric currents (ICs) produced by the FACs so that it is expressed as

$$
\mathrm{DPpi} / \mathrm{mi} .=\mathrm{DPpi} / \mathrm{mi}(\mathrm{FAC})+\mathrm{DPpi} / \mathrm{mi}(\mathrm{IC})
$$

The ionospheric equivalent current systems for the DPpiand DPmi-field show a twin vortex type caused by a dusk-to-dawn and dawn-to-dusk electric field, respectively. Tamao (1964) assumed that the high-latitude PI is produced by an Alfven mode HM wave incident to the polar ionosphere. The associated horizontal electric field is transmitted almost instantaneously to lower latitudes in the space below the ionosphere to cause low- and middle-latitude PIs (Kikuchi et al., 1978; Kikuchi and Araki, 1979).

The DPmi originates in the dawn-to-dusk convection electric field enhanced after the sudden magnetospheric

Copy right@ The Society of Geomagnetism and Earth, Planetary and Space Sciences (SGEPSS); The Seismological Society of Japan; The Volcanological Society of Japan; The Geodetic Society of Japan; The Japanese Society for Planetary Sciences; TERRAPUB. compression (Araki, 1977, 1994). The FACs and equivalent ionospheric current system for the DPmi-field is schematically illustrated in Fig. 1. The pattern of the equivalent current system for DPmi is assumed to be same as that for DPpi given in Araki (1994), but the current direction is reversed. If the location of footprints and distribution of the FAC are different from those for DPpi, the pattern will be slightly changed.

As the primary source of the DL-field is the enhanced magnetopause current, it is expected that the $H$-component amplitude $(\Delta H)$ of the main impulse (DLmi+DPmi) of SCs observed at low latitudes should be larger on the dayside than on the nightside. In actual fact, the SC amplitude of the northward magnetic field at the geosynchronous orbit shows a simple LT variation which takes the maximum around noon and minimum near midnight (Kokubun, 1983; Kuwashima et al., 1985). Observations on the ground, however, are opposite to this expectation. Ferraro and Unthank (1951) showed that the averaged $\Delta H$ of 55 SCs and 46 sudden impulses (SIs) observed at low- and middle-latitude stations is larger near midnight than in daytime. Russell et al. (1992, 1994) studied 18 and 7 SCs which occurred during the northward and southward interplanetary magnetic field (IMF), respectively, and reported that the amplitude is larger in the daytime during the northward IMF but it is reduced in the daytime and enhanced in the nighttime when the IMF is southward.

Araki et al. (2006) examined LT variations of the averaged $\Delta H$ of more than $600 \mathrm{SCs}$ at Memambetsu $\left(35.4^{\circ}\right.$ geomagnetic lat.), Kakioka $\left(27.4^{\circ}\right)$, Kanoya $\left(21.9^{\circ}\right)$, and Alibag $\left(10.2^{\circ}\right)$. Although Alibag shows a comparable amplitude at noon and midnight, the three Japanese stations obtain their maximum around midnight, with a secondary 

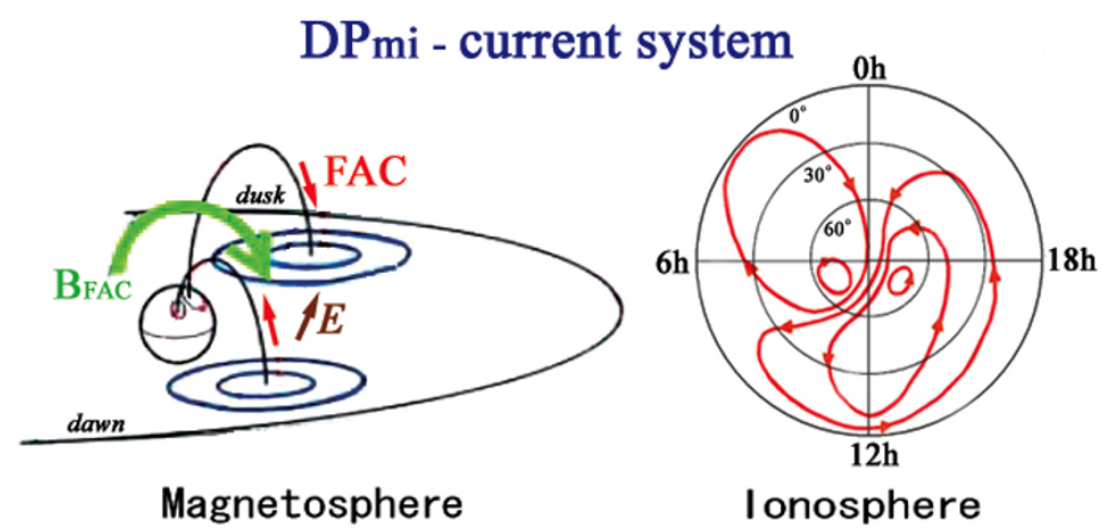

Fig. 1. Electric current system for the DPmi-field of SC. Left panel: FAC (red) shows field-aligned currents due to enhanced magnetospheric convection after a sudden compression of the magnetosphere. $B_{\mathrm{FAC}}$ (green) shows the FAC-produced magnetic field. Right panel: ionospheric equivalent current system for DPmi.

peak near noon and the minimum around 8 LT. They also checked the IMF dependence of the LT variation of $\Delta H$ and found that $\Delta H$ is larger in nighttime regardless of the IMF$B_{z}$ polarity but that the size of the LT variation becomes larger during the southward IMF. They interpreted the LT variation of the SC amplitude described above by a resultant magnetic field of the FAC that is responsible for DPmi and FAC-produced IC (denoted by $\mathrm{IC}_{\mathrm{FAC}}$ hereafter). Shinbori (private communication) confirmed these results by a statistical analysis of more than 2300 sudden magnetospheric compression events.

Kikuchi et al. (2001) analyzed the LT variation of the amplitude of positive PIs (PPIs) (Kikuchi and Araki, 1985) observed at low- and middle-latitude stations. Kikuchi et al. (2001) pointed out that the contribution of the magnetic fields due to the FAC for DPpi is important for the interpretation of PPI. These researchers calculated LT variations of the $H$-component PI amplitude due to both the FACs and $\mathrm{IC}_{\mathrm{FAC}}$ by giving a polar cap potential on the thin shell ionosphere with realistic conductivities, including LT and latitudinal variation and weak auroral enhancement.

The description above suggests that the FAC-produced magnetic field is an important consideration when interpreting the disturbance field of SCs even at low and middle latitudes.

In this paper we derive the LT variation of the averaged $H$-component amplitude of SC at Memanbetsu for the summer and winter seasons separately and compare it with calculations of the magnetic fields due to FACs for DPmi and $\mathrm{IC}_{\mathrm{FAC}}$. We then show a method to estimate both DLmiand DPmi-field and polar cap potential difference associated with the DPmi-field.

\section{Observed and Calculated LT Variation of the Averaged SC Amplitude}

The left panels of Fig. 2 show LT variations of the averaged $\Delta H$ of SCs observed at Memambetsu. The amplitude is taken from the SC list provided by the Kakioka Geomagnetic Observatory and averaged in each hourly bin. It was derived separately for the summer (upper panel) and winter (lower panel). The blue curve was obtained by taking a running average of $3 \mathrm{~h}$, and the red curve was obtained by taking a second running average of $3 \mathrm{~h}$. A total of $634 \mathrm{SCs}$ were observed in the 1957-2003 period; these are divided into four seasons, and so about one fourth of the events are used for each summer and winter season. Therefore, the curves in the left panels are not as smooth as the LT variation for the 634 SC events shown in Araki et al. (2006). The hourly standard deviation is $13-56 \mathrm{nT}$ in summer and 7-25 nT in winter, with a range of 16-37 nT for the LT variation of the total 634 events.

The following characteristics of the LT variation of $\Delta H$ can be determined from Fig. 2:

(A1) Both LT variations commonly show the maximum near midnight and the second maximum on the dayside. They also show the minimum at 7-8 $\mathrm{h} \mathrm{LT}$ and the second minimum in the evening.

(A2) The size of the LT variation is about $52 \mathrm{nT}$ in the summer and $16 \mathrm{nT}$ in the winter.

In two right panels of Fig. 2, we plotted calculated LT variations at $35^{\circ}$ geomagnetic latitude of the $H$-component magnetic field due to a pair of FACs and $\mathrm{IC}_{\mathrm{FAC}}$. The maximum FACs flow into (out from) the ionosphere at $7 \mathrm{~h}(17 \mathrm{~h}$ ) magnetic local time (MLT) and $75^{\circ}$ geomagnetic latitude. Realistic conductivities which depend upon LT and latitude and include weak auroral enhancement were given on the thin shell spherical ionosphere.

The intensity of the FACs was adjusted so as to produce a $100-\mathrm{kV}$ potential difference between their maximum points in the polar cap. In actual fact, these magnetic fields were obtained by reversing the sense of the calculated magnetic fields in Kikuchi et al. (2001) where they assumed FACs produced by a dusk-to-dawn magnetospheric electric field.

Based on the right panels we can derive the following statements:

(B1) In both summer and winter the IC-field reaches the maximum around noon and the negative minimum at 7$8 \mathrm{~h} \mathrm{LT}$. It is almost zero near midnight, reflecting low conductivities of the ionosphere. The FAC-field reaches the positive maximum at midnight and the negative minimum at noon.

(B2) The size of the LT variation of both IC- and FAC-field is larger in the summer than the winter.

(B3) The resultant magnetic field $(\mathrm{FAC}+\mathrm{IC})$ due to both 


\section{LT Variation of SC Amplitude (H-comp.)}
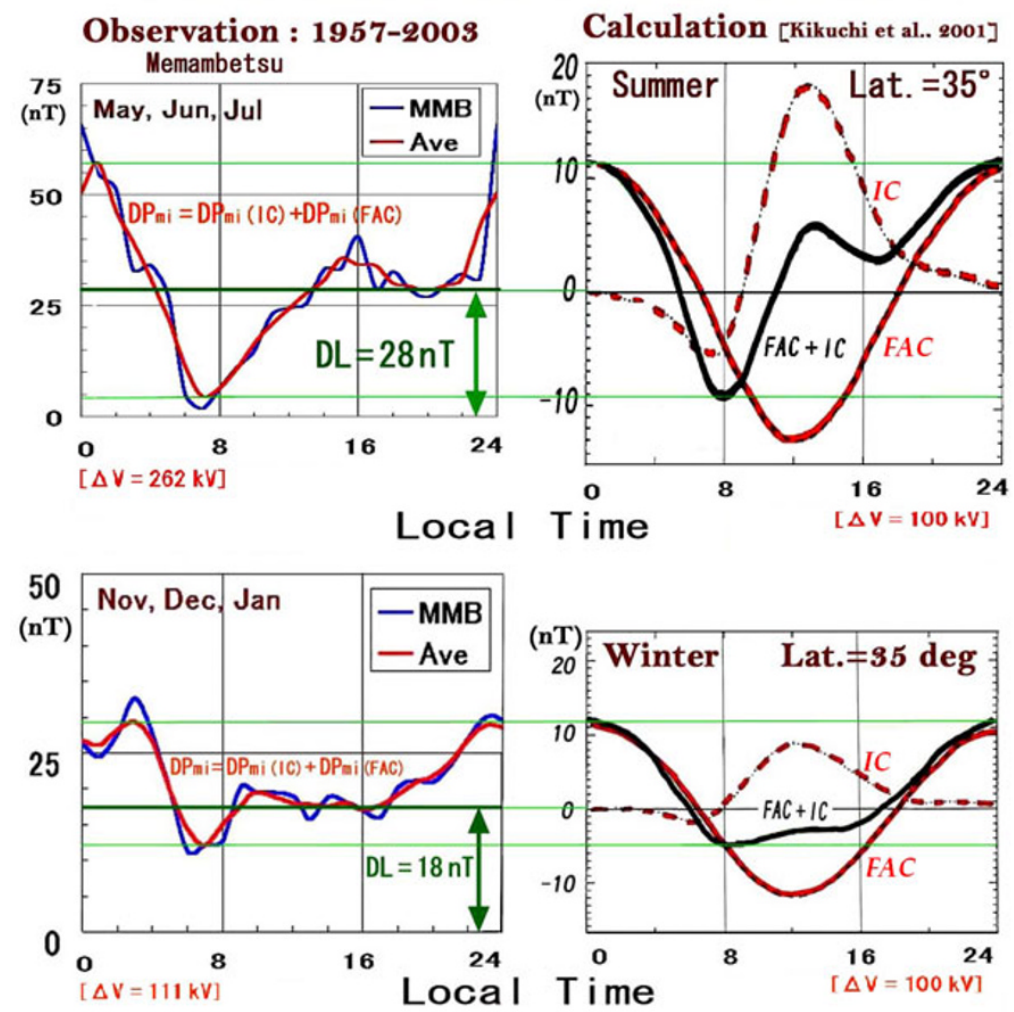

Fig. 2. LT variations of $H$-component amplitude. Upper panel: summer, lower panel: winter, left: SC observed at Memambetsu, right: calculation at $35^{\circ}$ geomagnetic latitude where FAC (red solid line), IC (red dashed line) and FAC+IC (black solid line) show $H$-component magnetic fields due to FAC, IC $\mathrm{FAC}_{\mathrm{F}}$, and both currents, respectively.

FAC and IC reaches the maximum near midnight, with a second peak near noon; the minimum is reached near $8 \mathrm{~h}$ LT, with a second minimum around $18 \mathrm{~h}$ LT.

(B4) The size of the LT variation of the resultant field is $20 \mathrm{nT}$ in the summer and $14 \mathrm{nT}$ in the winter.

By comparing the left and right panels in Fig. 2, we can say that the calculated LT variation agrees reasonably well with the observed one in both the summer and winter season although the LT of the maximum and minimum amplitude is slightly different.

\section{Estimation of the DLmi- and DPmi-field and Polar Cap Potential Difference Associated with DPmi}

Here, it is assumed that the DLmi-field does not depend upon LT so that the observed LT variation is due to the LT variation of the DPmi-field produced by the FAC and $\mathrm{IC}_{\mathrm{FAC}}$.

We adjusted the amplitude scale of the two left panels in Fig. 2 so that the maximum and minimum amplitude of the LT variation coincide with the calculated maximum and minimum amplitude in the two right panels, respectively. Then, comparing the left and right panels, we can determine the zero level of the LT variation of the observed DPmifield, as shown by thick green horizontal lines. This zero level of the DPmi-field corresponds to the observed DLmifield. By adopting this approach, we were able to obtain the DLmi-field as $28 \mathrm{nT}$ for summer and $18 \mathrm{nT}$ for winter.

The FAC used in the calculation in Fig. 2 is adjusted to produce a $100-\mathrm{kV}$ potential difference between the footprints of the maximum and minimum intensity. This FAC and $\mathrm{IC}_{\mathrm{FAC}}$ cause the LT variation of the resultant magnetic field to be of a magnitude of $20 \mathrm{nT}$ in the summer and $14 \mathrm{nT}$ in the winter. On the other hand, $52 \mathrm{nT}$ is found in the summer and $16 \mathrm{nT}$ in winter for the size of the LT variation of the averaged SC amplitude. If we assume that the observed LT variation is caused by the combined effects of the calculated $\mathrm{FAC}$ and $\mathrm{IC}_{\mathrm{FAC}}$, the polar cap potential difference due to the FACs should be $52 / 20 \times 100=260 \mathrm{kV}$ in the summer and $16 / 14 \times 100=113 \mathrm{kV}$ in the winter. These are the averaged potential differences which produce the LT variation of the averaged SC amplitude.

\section{Discussion}

The physical model of SC used here (Araki, 1977, 1994) is essentially consistent with the computer simulations of SC phenomena by Fujita et al. (2003a, b, 2005). This means that the decomposition of the Dsc into the DL- and DP-field is physically meaningful. The reasonable agreement between the calculations and observations in Fig. 2 also supports the validity of the model. It has not been possible, however, to estimate both the DL and DP field separately from the observed Dsc. A conventional approach is to treat low-latitude SCs as the pure DL-field, but it is not clear if the DP-field is negligibly small there. Here we have shown a method which can estimate each DLmi- and DPmi-field and the DPmi associated polar cap potential quantitatively 
by comparing the observed LT variation of the SC amplitude with the theoretical calculation based upon the proposed SC model.

Although the pattern of the calculated LT variations for both the summer and winter seasons is similar with the observed pattern on a whole, there is a difference between the calculated and observed LT of the maximum and minimum of the LT variations. Also, the polar cap potential difference obtained is much larger than that usually considered. These are primarily because the calculation in this analysis was not designed to interpret directly the observed LT variations described here. We utilized the results of Kikuchi et al. (2001) calculated for the DPpi by reversing the current direction. We will be able to obtain a better agreement between the observations and calculations by adjusting the parameters of the calculation (location and distribution of the FACs and ionospheric conductivities). Auroral enhancement of the conductivities strongly affects the potential difference in the polar cap. The calculation used here assumes a geomagnetic quiet state with a small auroral conductivity enhancement. If the enhancement is larger, the potential difference will become smaller. Moreover, we need more events in order to obtain a smoother LT variation of the observed SC amplitude.

It is physically important to study which of the constant voltage and current source fits with observations. In quantifying the agreement between the observed LT variations with the calculations for the two kinds of the sources, we will be able to obtain a solution of this problem.

Here, we have described the method under consideration by using SCs observed at one station, Memambetsu, but it is desirable to use data at several stations situated at different latitudes from the equator to higher latitudes and to select a combination of parameters of the calculation which best fits a set of the observations. Shinbori et al. (2009) has derived LT variations of averaged amplitude of magnetospheric compression events at six stations in the $0-45^{\circ}$ geomagnetic latitude range. These results can be used in future studies.

Although we have compared the calculated LT variation with that of averaged SC amplitude, the method is applicable for one particular SC if a reasonable LT variation of the amplitude of the SC is obtained. Araki et al. (2006) showed clear LT variations for two SCs which occurred during northward and southward IMF. Adjusting the parameters of the calculation to each SC, we will be able to know IMF-dependence of the FAC and $\mathrm{IC}_{\mathrm{FAC}}$ and the polar cap potential associated with the SC.

Through this analysis we recognized that the theoretical calculation described above is effective to study SC phenomena. The global distribution of ICs (including their daytime enhancement in the equator) due to a pair of FACs was calculated by Tsunomura and Araki (1984) at first. Osada (1992) evaluated LT and latitudinal dependence of magnetic fields due to the FAC and $\mathrm{IC}_{\mathrm{FAC}}$ separately for PI and MI. Tsunomura $(1998,1999)$ derived LT variations of the $H$ and $D$-component due to the FACs and ICs for different models for the FAC and ionospheric conductivities. Here, we have simply introduced a method for quantitative estimation of the DLmi- and DPmi-field and the associated potential difference. By refining the calculation and using more detailed observations for the LT variations at different latitudes, we will be able to obtain more physically reasonable results.

Acknowledgments. The authors thank Kakioka Geomagnetic Observatory, Japan Meteorological Agency for providing the SC list used in the analysis of this paper.

\section{References}

Araki, T., Global structure of geomagnetic sudden commencements, Planet. Space Sci., 25, 373-384, 1977.

Araki, T., A physical model of geomagnetic sudden commencement, Geophys. Monogr., 81, AGU, 183-200, 1994.

Araki, T., K. Keika, T. Kamei, H. Yang, and S. Alex, Nighttime enhancement of the amplitude of geomagnetic sudden commencement and its dependence on IMF-Bz, Earth Planets Space, 58, 45-50, 2006.

Ferraro, V. C. A. and H. W. Unthank, Sudden commencements and sudden impulses in geomagnetism: their diurnal variation in amplitude, Geofis. Pure Appl., 20, 2730, 1951.

Fujita, S., T. Tanaka, T. Kikuchi, K. Fujimoto, K. Hosokawa, and M. Itonaga, A numerical simulation of the geomagnetic sudden commencement: 1 . Generation of the field-aligned current associated with the preliminary impulse, J. Geophys. Res., 108(A12), 1416, doi:10. 1029/2002JA009407, 2003a.

Fujita, S., T. Tanaka, T. Kikuchi, K. Fujimoto, and M. Itonaga, A numerical simulation of the geomagnetic sudden commencement: 2. Plasma processes in the main impulse, J. Geophys. Res., 108(A12), 1417, doi:10. 1029/2002JA009763, 2003b.

Fujita, S., T. Tanaka, and T. Motoba, A numerical simulation of the geomagnetic sudden commencement: 3 . A sudden commencement in the magnetosphere-ionosphere compound system, J. Geophys. Res., 110, doi:10.1029/2005JA011055, 2005.

Kikuchi, T. and T. Araki, Horizontal transmission of the polar electric field, J. Atmos. Terr. Phys., 41, 927-936, 1979.

Kikuchi, T. and T. Araki, Preliminary positive impulse of geomagnetic sudden commencement observed at dayside middle and low latitudes, J. Geophys. Res., 90, 12195-12200, 1985.

Kikuchi, T., T. Araki, K. Maekawa, and H. Maeda, Transmission of polar electric field to the equator, Nature, 273, 650-651, 1978.

Kikuchi, T., S. Tsunomura, K. Hashimoto, and K. Nozaki, Field-alinged current effects on mid-latitude geomagnetic sudden commencements, $J$. Geophys. Res., 106, 15555-15565, 2001.

Kokubun, S., Characteristics of storm sudden commencement at geostationary orbit, J. Geophys. Res., 88, 10025-10033, 1983.

Kuwashima, M., S. Tsunomura, and H. Fukunishi, SSC associated magnetic variations at the geosynchronous altitude, J. Atmos. Terr. Phys., 47, 451-461, 1985.

Osada, S., Numerical calculation of the geomagnetic sudden commencement, Master's Thesis, Faculty of Science, Kyoto University, 1992.

Russell, C. T., M. Ginskey, S. Petrinec, and G. Le, The effects of solar wind dynamic pressure changes on low and mid-latitude magnetic records, Geophys. Res. Lett., 19, 1227-1230, 1992.

Russell, C. T., M. Ginskey, and S. Petrinec, Sudden impulses at low latitude stations: Steady state response for southward interplanetary magnetic field, J. Geophys. Res., 99, 13403-13408, 1994.

Shinbori, A., Y. Tsuji, T. Kikuchi, T. Araki, and S. Watari, Magnetic latitude and local time dependence of the amplitude of geomagnetic sudden commencement, J. Geophys. Res., 2009 (accepted).

Tamao, T., Hydromagnetic interpretation of geomagnetic ssc*, Rep. Ionosp. Space Res. Japan, 18, 16-31, 1964.

Tsunomura, S., Characteristics of geomagnetic sudden commencement observed in middle and low latitudes, Earth Planets Space, 50, 755$772,1998$.

Tsunomura, S., Numerical analysis of global ionospheric current system including the effect of equatorial enhancement, Ann. Geophys., 17, 692706, 1999.

Tsunomura, S. and T. Araki, Numerical analysis of equatorial enhancement of geomagnetic sudden commencement, Planet. Space Sci., 32, 599-604, 1984.

T. Araki (e-mail: t.araki@kw2.ecs.kyoto-u.ac.jp), S. Tsunomura, and T. Kikuchi 